\title{
Managing frailty in primary care: evidence gaps cannot be ignored
}

\author{
Sathya Karunananthan PhD, Howard Bergman MD
}

Cite as: CMAJ 2018 September 24;190:E1122-3. doi: 10.1503/cmaj.181151

See related article at www.cmaj.ca/lookup/doi/10.1503/cmaj.171509

M

ore than two decades have passed since researchers set out to provide scientific evidence for the clinical concept of frailty. Physicians had always recognized that some older adults exhibited a certain vulnerability. Although they knew "frailty" when they saw it, physicians struggled to define it. Through secondary analyses of large data sets and expert meetings, consensus emerged that frailty is a state of vulnerability, arising from impairments in multiple organ systems, leading to increased susceptibility to poor health outcomes. ${ }^{1}$ But the consensus ended there. In a linked article, Abbasi and colleagues advocate for frailty case finding in the primary care setting. ${ }^{1}$ However, lack of consensus on a clinical definition or tool for identifying frailty, and lack of evidence on the clinical utility of its detection, remain important barriers to identifying and managing frailty in primary care.

Leading geriatricians and gerontologists stated in 2006 that "In the land of frailty, confusion, contradiction and ambiguity reign supreme."2 Although research in this area has greatly increased in the last decade, efforts to define and operationalize frailty have continued to generate conflicting ideas of the concept, its measurement and its relationships with aging, disability and chronic disease. Whereas the early research on frailty aimed to identify vulnerable older adults before the onset of disability and beyond the usual limitations of chronic disease, many tools for recognizing frailty have included disability and chronic diseases as criteria for frailty. A recent review of frailty assessment instruments showed substantial heterogeneity across 67 scores in the identification of individuals as frail. ${ }^{3}$ The single most widely adopted "frailty phenotype," first described by Fried and colleagues in 2001, ${ }^{4}$ has been operationalized in 262 different ways. ${ }^{5}$

Definitions and tools for frailty born out of secondary analyses of data from studies of aging ${ }^{3}$ have provided clear evidence that individuals who meet the criteria for frailty, however it is defined, are at higher risk of medical complications, disability, institutionalization or even death, compared with those who are not frail. ${ }^{6}$ Such statistical associations, however robust, do not provide a sound justification for frailty case finding; risk factors identified at the population level may not be good predictors of outcomes at the patient level. Indeed, the few studies that have assessed the

\section{KEY POINTS}

- There is currently no consensus on a definition or clinical tool to identify frailty, and insufficient evidence to guide frailty case finding and management in primary care.

- Although studies have shown that medical complications, disability, institutionalization or even death are more likely among frail than non-frail groups, evidence is lacking on how well frailty predicts adverse outcomes at the individual patient level.

- Screening for frailty in the primary care setting will be justifiable only when adequate evidence of interventions that improve clinical and patient-centred outcomes emerges.

predictive ability of frailty markers suggest that they contribute very little to the prediction of adverse health outcomes. ${ }^{\text {? }}$

The utility of case finding for frailty in primary care is further hindered by the current lack of suitable evidence to inform clinical decision-making. ${ }^{8,9}$ Although physical activity, nutrition, memory training and the comprehensive geriatric assessment have been explored as single or multicomponent interventions, ${ }^{9}$ none of these are specific to frailty. The comprehensive geriatric assessment was developed to address disability in older adults ${ }^{10}$ well before the emergence of the concept of frailty in the scientific literature. As interventions such as the Mediterranean diet and increased physical activity have been suggested for preventing and managing some chronic diseases that are highly prevalent in older populations, researchers must show that these interventions have added value when targeted to frail patients. As yet, there is a lack of evidence that they reduce frailty, let alone improve clinical and patient-centred outcomes in frail patients. ${ }^{8,9,11}$

Costs and potential harms related to the clinical use of frailty tools in routine practice also warrant consideration. Canadian primary care providers face severe time and resource constraints. In the absence of a clear definition and evidence for clinical decision-making, costs related to screening for frailty in primary care are unlikely to translate into improved clinical outcomes. Frailty research has also not generally considered patient perspectives. Older patients have expressed disagreement with researchers' definitions of frailty and object to being 
labelled as "frail." "8,10 Screening for frailty can do more harm than good if it stigmatizes patients. Future research and development of clinical decision-making aids related to frailty cannot take place without the engagement of older patients. ${ }^{12}$

Evidence-based guidance to support screening and management of frailty in primary care is sorely needed. Current priorities are to establish consensus on a clinical tool that provides added clinical value, and to assess whether interventions improve patient-centred outcomes. Until these priorities have been addressed, identifying frailty in primary care may lead to unwarranted costs and patients may experience inadvertent harms.

\section{References}

1. Abbasi M, Rolfson D, Khera AS, et al. Identification and management of frailty in the primary care setting. CMAJ 2018;190:E1134-40.

2. Ferrucci L, Mahallati A, Simonsick EM. Frailty and the foolishness of Eos. $J$ Gerontol A Biol Sci Med Sci 2006;61:260-1.

3. Buta BJ, Walston JD, Godino JG, et al. Frailty assessment instruments: systematic characterization of the uses and contexts of highly-cited instruments. Ageing Res Rev 2016;26:53-61.

4. Fried LP, Tangen CM, Walston J, et al.; Cardiovascular Health Study Collaborative Research Group. Frailty in older adults: evidence for a phenotype. J Gerontol A Biol Sci Med Sci 2001;56:M146-56.

5. Theou O, Cann L, Blodgett J, et al. Modifications to the frailty phenotype criteria: Systematic review of the current literature and investigation of 262 frailty phenotypes in the Survey of Health, Ageing, and Retirement in Europe. Ageing Res Rev 2015;21:78-94.

6. Shamliyan T, Talley KM, Ramakrishnan R, et al. Association of frailty with survival: a systematic literature review. Ageing Res Rev 2013;12:719-36.
7. Sourial N, Bergman H, Karunananthan S, et al. Implementing frailty into clinical practice: a cautionary tale. J Gerontol A Biol Sci Med Sci 2013;68:1505-11.

8. Hogan DB, Maxwell CJ, Afilalo J, et al. A scoping review of frailty and acute care in middle-aged and older individuals with recommendations for future research. Can Geriatr J 2017;20:22-37.

9. Walston J, Buta B, Xue QL. Frailty screening and interventions: considerations for clinical practice. Clin Geriatr Med 2018;34:25-38.

10. Puts MTE, Toubasi S, Andrew MK, et al. Interventions to prevent or reduce the level of frailty in community-dwelling older adults: a scoping review of the literature and international policies. Age Ageing 2017;46:383-92.

11. Parker SG, McCue P, Phelps K, et al. What is comprehensive geriatric assessment (CGA)? An umbrella review. Age Ageing 2018;47:149-55.

12. Holroyd-Leduc J, Resin J, Ashley L, et al. Giving voice to older adults living with frailty and their family caregivers: engagement of older adults living with frailty in research, health care decision making, and in health policy. Res Involv Engagem 2016;2:23.

\section{Competing interests: None declared.}

This article was solicited and has not been peer reviewed.

Affiliations: Clinical Epidemiology Program (Karunananthan), Ottawa Hospital Research Institute, Ottawa, Ont.; Department of family Medicine (Bergman), McGill University, Montréal, Que.

Contributors: Both authors contributed to the conception and design of the work, drafted the manuscript, revised it critically for important intellectual content, gave final approval of the version to be published and agreed to be accountable for all aspects of the work.

Correspondence to: Howard Bergman, howard.bergman@mcgill.ca 